TITLE:

\title{
Investigation of ultrafast nuclear spin polarization induced by short laser pulses
}

$\operatorname{AUTHOR}(S)$ :

Nakajima, T

CITATION:

Nakajima, T. Investigation of ultrafast nuclear spin polarization induced by short laser pulses. Physical Review Letters 2007, 99(2): 024801.

ISSUE DATE:

2007-07-13

URL:

http://hdl.handle.net/2433/50428

RIGHT:

Copyright 2007 American Physical Society 


\title{
Investigation of Ultrafast Nuclear Spin Polarization Induced by Short Laser Pulses
}

\author{
Takashi Nakajima* \\ Institute of Advanced Energy, Kyoto University, Gokasho, Uji, Kyoto 611-0011, Japan
}

(Received 6 November 2006; published 12 July 2007)

\begin{abstract}
We theoretically investigate the dynamics of nuclear spin induced by short laser pulses and show that ultrafast nuclear spin polarization can take place. Combined use of the hyperfine interaction together with the static electric field is the key for that. Specifically we apply the idea to unstable isotopes, ${ }^{27} \mathrm{Mg}$ and ${ }^{37} \mathrm{Ca}$, with nuclear spin of $1 / 2$ and $3 / 2$, respectively, and show that $88 \%$ and $62 \%$ of nuclear spin polarization can be achieved within a few to tens of ns, which is $2-3$ orders of magnitude shorter than the time needed for any known optical methods. Because of its ultrafast nature, our scheme would be very effective not only for stable nuclei but also unstable nuclei with a lifetime as short as $\mu$ s.
\end{abstract}

The need for spin-polarized nuclei extends from fundamental nuclear physics [1] to medical applications such as nuclear magnetic resonance. Related to the latter, laser production of spin-polarized Xe gas was first reported in Ref. [2]. Because of the great demand for spin-polarized nuclei, several methods have been proposed and investigated so far, some of which include the use of the angleand mass-resolved secondary beam after nuclear fragmentation at a target, optical pumping [3], and the combination of optical pumping and spin-exchange collisions in a buffer gas $[2,4]$. Although the use of the secondary beam mentioned above is very useful to obtain spin-polarized nuclei, the achievable degree of polarization is very low $(<1 \%)$. In contrast, the optical pumping method works very well for alkali atoms such as $\mathrm{Li}$ and enables us to obtain the high degree of spin polarization [3], the major drawback being the complicated optimization in terms of the multiple laser wavelengths and bandwidth which are to be matched, with the use of electro-optic modulators, to the Dopplerbroadened multiple hyperfine transition lines. In addition, due to the need of a continuous wave laser with a moderately high laser power, the available wavelength is limited to the visible range. Combined use of optical pumping and spin-exchange collisions gives us some compromise between the two methods mentioned above and is applicable to various nuclei at the expense of further complications and long interaction time needed to attain high degree of spin polarization because it is an indirect method. Moreover, as long as one utilizes optical pumping in one way or another, at least a few $\mu$ s interaction time is necessary until nuclear spin polarization has been completed. This is a fundamental limitation of the known optical methods to polarize unstable nuclei with short lifetimes.

Related to nuclear spin, polarized electrons and electron-spin-polarized ions, etc. are also known to be very useful to study various kinds of spin-dependent phenomena. For example, polarized electrons [5] are highly needed in high-energy physics, while polarized electrons and electron-spin-polarized ions, including neutral atoms and molecules, are useful in surface physics [6] and atomic and molecular physics [7]. Recently we have theoretically as well as experimentally investigated the control of spin polarization of electrons and ions using laser pulses [810]. In particular, use of the pump and probe laser pulses with short pulse duration has turned out to be a new doorknob to control spin degree of freedom [9-11].

In this Letter, we theoretically investigate the dynamics of nuclear spin polarization in atomic systems using short laser pulses and demonstrate that our scenario is very effective for alkaline-earth atoms such as $\mathrm{Be}, \mathrm{Mg}, \mathrm{Ca}, \mathrm{Sr}$, $\mathrm{Ba}$, and also alkaline-earth-like ions such as $\mathrm{B}^{+}, \mathrm{Al}^{+}, \mathrm{Ga}^{+}$, $\mathrm{In}^{+}$, and $\mathrm{Tl}^{+}$. There are three main advantages for our scheme. The first advantage is that the time needed for nuclear spin polarization is surprisingly short, and only a few to tens of ns is sufficient to polarize nuclei, which is $2-3$ orders of magnitude shorter compared with the time needed for any known optical methods. Therefore, our scheme may be efficiently applied to even unstable nuclei with short lifetimes. The key of such ultrafast nuclear spin polarization is to create a coherent superposition of hyperfine manifolds in excited states using a short laser pulse and let them evolve in time through hyperfine interaction. The time scale of hyperfine interaction, however, is usually very long since it is inherently very weak. Naturally spontaneous decay will take place before nuclear spin polarization has been completed, and our scheme does not seem to work. We will show that we could overcome this fundamental problem by the introduction of a static electric field. The second advantage is that, since we employ a pulsed laser, the available wavelength extends from the infrared to the vacuum ultraviolet. This gives us far more flexibility compared with the optical pumping method. The third advantage is that we do not have to worry about the Doppler broadening of the transition line, since the laser bandwidth is already broader than that. After the derivation of the general equations, we present specific numerical results for unstable isotopes, ${ }^{27} \mathrm{Mg}$ and ${ }^{37} \mathrm{Ca}$, with nuclear spin of $1 / 2$ and $3 / 2$, respectively, and show that, despite its simplicity, nuclear spin polarization as high as $88 \%$ and $62 \%$, respectively, can be obtained in the ultrafast time 
scale. We would like to emphasize that our scheme is reasonably general and applicable to the isotopes of any alkaline-earth atoms with nuclear spin of $1 / 2$ and $3 / 2$.

For the maximum clarity, we start a discussion for the ${ }^{27} \mathrm{Mg}$ atom with $I=1 / 2$ where $I$ stands for the nuclear spin. The scheme we propose consists of the ground state, $3 s^{2}{ }^{1} S_{0}$, and the excited state, $3 s 6 p{ }^{1} P_{1}$ with an initial condition that all magnetic sublevels of the ground state are equally populated. By taking into account the nuclear spin, the total angular momentum, $F$, of the ground state becomes $F=1 / 2$, while the excited state splits into the two hyperfine sublevels with $F=1 / 2$ and $3 / 2$. For the purpose of what follows, it is essential to consider the dynamics for all different magnetic sublevels, $m_{F}$, separately as shown in Fig. 1(a), in terms of the coupled basis description, $\left|(J I) F m_{F}\right\rangle$. To summarize, we define $|0\rangle=\mid\left(3 s^{21} S_{0}\right) F=$ $\left.1 / 2 m_{F}=-1 / 2\right\rangle, \quad|1\rangle=\left|\left(3 s 6 p^{1} P_{1}\right) F=1 / 2 m_{F}=1 / 2\right\rangle$, $|2\rangle=\left|\left(3 s 6 p^{1} P_{1}\right) F=3 / 2 m_{F}=1 / 2\right\rangle, \quad|3\rangle=\mid\left(3 s^{21} S_{0}\right) F=$ $\left.1 / 2 m_{F}=1 / 2\right\rangle$, and $|4\rangle=\left|\left(3 s 6 p^{1} P_{1}\right) F=3 / 2 m_{F}=3 / 2\right\rangle$.

We now introduce a sub-ns laser pulse at the wavelength of $174.8 \mathrm{~nm}$ with right-circular polarization to coherently excite hyperfine manifolds of $3 s 6 p{ }^{1} P_{1}$, i.e., $|1\rangle$ and $|2\rangle$, which is possible since the hyperfine energy splitting is smaller than the spectral bandwidth of the sub-ns laser pulse. From the detailed time-dependent analysis together with atomic structure calculations, we have found that the intensity of this laser is as low as $\sim 2.4 \mathrm{~kW} / \mathrm{cm}^{2}$ assuming a 100 ps pulse.

For a better understanding of the time-dependent behavior of nuclear spin, it is more convenient to employ the uncoupled basis description, $\left|\left(\operatorname{Jm}_{J}\right)\left(\operatorname{Im}_{I}\right)\right\rangle \equiv\left|J m_{J}\right\rangle\left|I m_{I}\right\rangle$, as shown in Fig. 1(b). For simplicity, we abbreviate $\left|I m_{I}\right\rangle$ to $\left|m_{I}\right\rangle$. The advantage of using the uncoupled basis set is that the quantum numbers for nuclear spin, $\left|\operatorname{Im}_{I}\right\rangle$, has been decoupled from the other quantum numbers, $\left|J m_{J}\right\rangle$, so that we can clearly see how the flip of nuclear spin takes place. To summarize, we define $\left|0^{\prime}\right\rangle=\left|3 s^{21} S_{0} m_{J}=0\right\rangle \mid m_{I}=$ $-1 / 2\rangle, \quad\left|1^{\prime}\right\rangle=\left|3 s 6 p^{1} P_{1} m_{J}=1\right\rangle\left|m_{I}=-1 / 2\right\rangle, \quad\left|2^{\prime}\right\rangle=$ $\left|3 s 6 p^{1} P_{1} m_{J}=0\right\rangle\left|m_{I}=1 / 2\right\rangle, \quad\left|3^{\prime}\right\rangle=\mid 3 s^{21} S_{0} m_{J}=$ $0\rangle\left|m_{I}=1 / 2\right\rangle$, and $\left|4^{\prime}\right\rangle=\left|3 s 6 p^{1} P_{1} m_{J}=1\right\rangle\left|m_{I}=1 / 2\right\rangle$.

Because of the very small hyperfine energy splittings a coherent superposition is easily created by the sub-ns laser pulse. Therefore, starting from $|0\rangle\left(=\left|0^{\prime}\right\rangle\right)$, the wave function of the excited hyperfine manifold specified by $m_{F}=$ $1 / 2$ is written, in the coupled basis, as
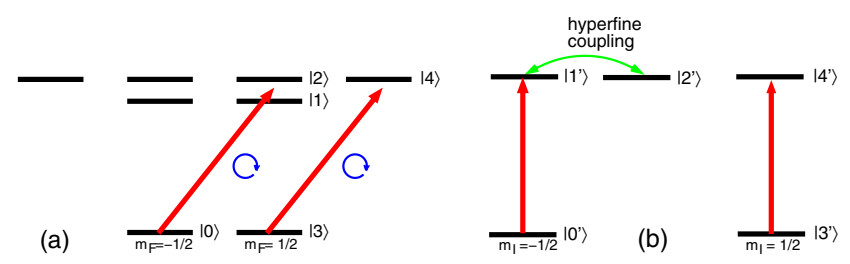

FIG. 1 (color online). Level scheme for the $I=1 / 2$ system of alkaline-earth atoms in the (a) coupled basis and (b) uncoupled basis descriptions.

$$
\psi_{\mathrm{ex}}^{m_{F}=1 / 2}(t)=\frac{\sqrt{2}}{3}|1\rangle e^{-i E_{1} t}+\frac{1}{3}|2\rangle e^{-i E_{2} t},
$$

where the superscript of $\psi$ represents $m_{F}$ of the excited hyperfine manifold, and $E_{k}(k=1,2, \ldots)$ stands for the energy of state $|k\rangle$ with respect to the ground state. Recalling that the coupled and uncoupled basis are connected through the relation of

$$
\left(\begin{array}{l}
\left|1^{\prime}\right\rangle \\
\left|2^{\prime}\right\rangle
\end{array}\right)=\left(\begin{array}{cc}
\sqrt{2 / 3} & 1 / \sqrt{3} \\
-1 / \sqrt{3} & \sqrt{2 / 3}
\end{array}\right)\left(\begin{array}{l}
|1\rangle \\
|2\rangle
\end{array}\right)
$$

we find that Eq. (1) can be rewritten as

$$
\begin{aligned}
\psi_{\mathrm{ex}}^{m_{F}=1 / 2}(t)= & \frac{1}{3 \sqrt{3}}\left(2 e^{-i E_{1} t}+e^{-i E_{2} t}\right)\left|1^{\prime}\right\rangle \\
& +\frac{\sqrt{2}}{3 \sqrt{3}}\left(-e^{-i E_{1} t}+e^{-i E_{2} t}\right)\left|2^{\prime}\right\rangle .
\end{aligned}
$$

As for the excitation from $|3\rangle\left(=\left|3^{\prime}\right\rangle\right)$ to $|4\rangle$,

$$
\psi_{\mathrm{ex}}^{m_{F}=3 / 2}(t)=\frac{1}{\sqrt{3}}\left|4^{\prime}\right\rangle e^{-i E_{4} t},
$$

since $\left|4^{\prime}\right\rangle=|4\rangle$. The corresponding physical picture for both paths starting from $\left|0^{\prime}\right\rangle$ and $\left|3^{\prime}\right\rangle$ is shown in Fig. 1(b) using the uncoupled basis. By making use of the formula for populations in each nuclear spin state, $\left|m_{I}= \pm 1 / 2\right\rangle$, i.e.,

$$
P_{m_{I}}(t)=\sum_{J, m_{J}} \sum_{m_{F}} \mid\left\langle(L S) J m_{J}\left|\left\langle m_{I} \mid \psi_{\mathrm{ex}}^{m_{F}}(t)\right\rangle\right|^{2},\right.
$$

we obtain

$$
P_{m_{I}=-1 / 2}(t)=\frac{1}{27}(5+4 \cos \Delta E t)
$$

and

$$
P_{m_{I}=1 / 2}(t)=\frac{4}{27}(1-\cos \Delta E t)+\frac{1}{3},
$$

where $\Delta E \equiv E_{1}-E_{2}$. Finally the degree of nuclear spin polarization is computed using

$$
P(t)=\frac{\sum_{m_{I}} m_{I} P_{m_{I}}(t)}{I \sum_{m_{I}} P_{m_{I}}(t)} .
$$

What we find from these equations is as follows. At the moment of laser excitation, $t=0$, the degree of spin polarization is zero as it should be, since nuclear spin does not play any role for the photoabsorption process. After the pulse, however, transient nuclear spin polarization starts to take place between $\left|1^{\prime}\right\rangle$ and $\left|2^{\prime}\right\rangle$ due to the hyperfine coupling, as shown in Fig. 1(b), with the period given by $(\Delta E)^{-1}$, and the possible maximum value is $P=$ $8 / 9$. However, we should not forget that the natural lifetime of the excited states of neutral atoms is typically in the range of a few to a few hundred ns. The hyperfine splittings of excited neutral atoms are typically of the order of a few MHz to sub-MHz, implying that $(\Delta E)^{-1}$ is of the order of a few $\mu$ s. Naturally, laser-excited atoms will decay to the lower states before the sufficient hyperfine coupling. 
Apparently, our method does not seem to work for real atoms.

In order to overcome this fundamental problem, we introduce a static electric field which induces dc stark shifts. Since the amount of dc stark shifts is state dependent [10] essentially due to the different angular momentum couplings, the energy intervals between hyperfine sublevels become a function of static electric field, i.e., $E_{k} \rightarrow$ $E_{k}+\delta_{k}$, where $\delta_{k}$ is a state-dependent dc stark shift. For the quantitative estimation of $\delta_{k}$, we have performed Hartree-Fock calculations for $\mathrm{Mg}$ by fully taking into account configuration interactions with more than 1000 electronic configurations for each symmetry, and we found that a few $\mathrm{kV} / \mathrm{cm}$ static electric field would be sufficient to accelerate the hyperfine interaction by $2-3$ orders of magnitude. Figure 2 shows the variation of $P(t)$ after the laser pulse. Because of the applied electric field, the modulation period is as fast as $50 \mathrm{~ns}$. By noting that the state-dependent dc stark shifts originate from the different angular coefficients for different hyperfine sublevels, it should be clear that the same temporal behavior is expected at different electric field strengths only if the time scale is properly changed.

Having demonstrated that our scheme works very well for $I=1 / 2$, we now consider the case of $I=3 / 2$. In particular, we consider the $4 s^{2}{ }^{1} S_{0}-4 s 6 p{ }^{1} P_{1}$ system of ${ }^{37} \mathrm{Ca}$ with an initial condition that all magnetic sublevels of the ground state are equally populated. Now the level scheme becomes much more complicated as shown in Fig. 3, since there are four transition paths and three hyperfine sublevels. Accordingly, there is no clear picture for $I=3 / 2$, and it is far from obvious whether our scheme works well. After some algebra the wave functions of each
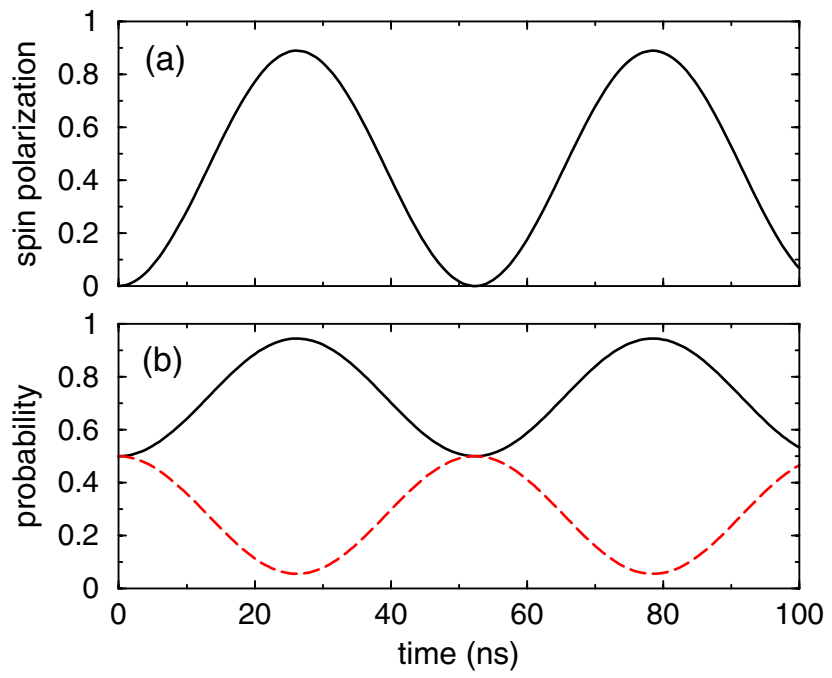

FIG. 2 (color online). Variation of the (a) degree of nuclear spin polarization and (b) probability in each nuclear spin state as a function of time for the ${ }^{27} \mathrm{Mg} 3 s^{2}{ }^{1} S_{0}-3 s 6 p{ }^{1} P_{1}(I=1 / 2)$ system under the application of a $3 \mathrm{kV} / \mathrm{cm}$ static electric field. In graph (b), solid and dashed lines represent probabilities in the $m_{I}= \pm 1 / 2$ nuclear spin states, respectively. hyperfine manifold are derived, in the uncoupled basis, as

$$
\begin{aligned}
\psi_{\mathrm{ex}}^{m_{F}=-1 / 2}(t)= & \left(\frac{\sqrt{3}}{6} e^{-i E_{1} t}+\frac{2 \sqrt{3}}{15} e^{-i E_{2} t}+\frac{\sqrt{3}}{30} e^{-i E_{3} t}\right)\left|1^{\prime}\right\rangle \\
& +\left(-\frac{\sqrt{2}}{6} e^{-i E_{1} t}+\frac{\sqrt{2}}{15} e^{-i E_{2} t}+\frac{\sqrt{2}}{10} e^{-i E_{3} t}\right)\left|2^{\prime}\right\rangle \\
& +\left(\frac{1}{6} e^{-i E_{1} t}-\frac{4}{15} e^{-i E_{2} t}+\frac{1}{10} e^{-i E_{3} t}\right)\left|3^{\prime}\right\rangle,
\end{aligned}
$$

$$
\begin{aligned}
\psi_{\mathrm{ex}}^{m_{F}=1 / 2}(t)= & \left(\frac{\sqrt{3}}{18} e^{-i E_{5} t}+\frac{8 \sqrt{3}}{45} e^{-i E_{6} t}+\frac{\sqrt{3}}{10} e^{-i E_{7} t}\right)\left|5^{\prime}\right\rangle \\
& +\left(-\frac{\sqrt{6}}{18} e^{-i E_{5} t}-\frac{2 \sqrt{6}}{45} e^{-i E_{6} t}+\frac{\sqrt{6}}{10} e^{-i E_{7} t}\right)\left|6^{\prime}\right\rangle \\
& +\left(\frac{1}{6} e^{-i E_{5} t}-\frac{4}{15} e^{-i E_{6} t}+\frac{1}{10} e^{-i E_{7} t}\right)\left|7^{\prime}\right\rangle,
\end{aligned}
$$

$$
\begin{aligned}
\psi_{\mathrm{ex}}^{m_{F}=3 / 2}(t)= & \left(\frac{2 \sqrt{3}}{15} e^{-i E_{9} t}+\frac{\sqrt{3}}{5} e^{-i E_{10} t}\right)\left|9^{\prime}\right\rangle \\
& +\left(-\frac{\sqrt{2}}{5} e^{-i E_{9} t}+\frac{\sqrt{2}}{5} e^{-i E_{10} t}\right)\left|10^{\prime}\right\rangle,
\end{aligned}
$$

$$
\psi_{\mathrm{ex}}^{m_{F}=5 / 2}(t)=\frac{1}{\sqrt{3}} e^{-i E_{12} t}\left|12^{\prime}\right\rangle,(12)
$$

where $\quad\left|0^{\prime}\right\rangle=\left|4 s^{21} S_{0} m_{J}=0\right\rangle\left|m_{I}=-3 / 2\right\rangle, \quad\left|1^{\prime}\right\rangle=$ $\left|4 s 6 p^{1} P_{1} m_{J}=1\right\rangle\left|m_{I}=-3 / 2\right\rangle,\left|2^{\prime}\right\rangle=\left|4 s 6 p^{1} P_{1} m_{J}=0\right\rangle \times$ $\left|m_{I}=-1 / 2\right\rangle,\left|3^{\prime}\right\rangle=\left|4 s 6 p^{1} P_{1} m_{J}=-1\right\rangle\left|m_{I}=1 / 2\right\rangle,\left|4^{\prime}\right\rangle=$ $\left|4 s^{21} S_{0} m_{J}=0\right\rangle\left|m_{I}=-1 / 2\right\rangle, \quad\left|5^{\prime}\right\rangle=\left|4 s 6 p^{1} P_{1} m_{J}=1\right\rangle \times$ $\left|m_{I}=-1 / 2\right\rangle,\left|6^{\prime}\right\rangle=\left|4 s 6 p^{1} P_{1} m_{J}=0\right\rangle\left|m_{I}=1 / 2\right\rangle,\left|7^{\prime}\right\rangle=$ $\left|4 s 6 p^{1} P_{1} m_{J}=-1\right\rangle\left|m_{I}=3 / 2\right\rangle,\left|8^{\prime}\right\rangle=\left|4 s^{21} S_{0} m_{J}=0\right\rangle \times$ $\left|m_{I}=1 / 2\right\rangle,\left|9^{\prime}\right\rangle=\left|4 s 6 p^{1} P_{1} m_{J}=1\right\rangle\left|m_{I}=1 / 2\right\rangle,\left|10^{\prime}\right\rangle=$ $\left|4 s 6 p^{1} P_{1} m_{J}=0\right\rangle\left|m_{I}=3 / 2\right\rangle, \quad\left|11^{\prime}\right\rangle=\left|4 s^{21} S_{0} m_{J}=0\right\rangle \times$ $\left|m_{I}=3 / 2\right\rangle$, and $\left|12^{\prime}\right\rangle=\left|4 s 6 p^{1} P_{1} m_{J}=1\right\rangle\left|m_{I}=3 / 2\right\rangle$. By making use of Eqs. (5) and (8), we obtain $P_{m_{I}}(t)$ for all possible $m_{I}$ and $P(t)$. We have performed specific numerical calculations with the static electric field of $3 \mathrm{kV} / \mathrm{cm}$. The result is shown in Fig. 4. Maximum spin polarization of $62 \%$ is reached at $5 \mathrm{~ns}$. It is clear now that our scheme is very effective for both $I=1 / 2$ and $3 / 2$ systems, and nuclear spin polarization can be achieved within a surprisingly short time scale.

Now, the remaining question is how to freeze nuclear spin polarization. This is a very important question, since

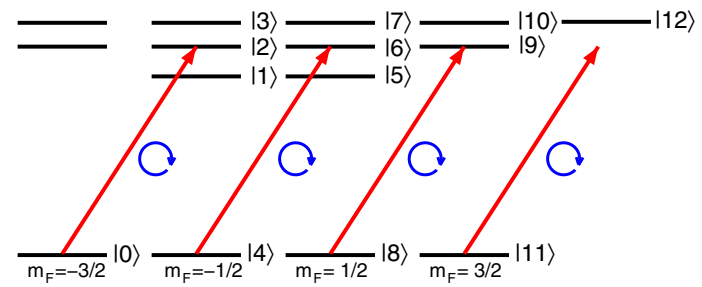

FIG. 3 (color online). Level scheme for the $I=3 / 2$ system of alkaline-earth atoms in the coupled basis. 

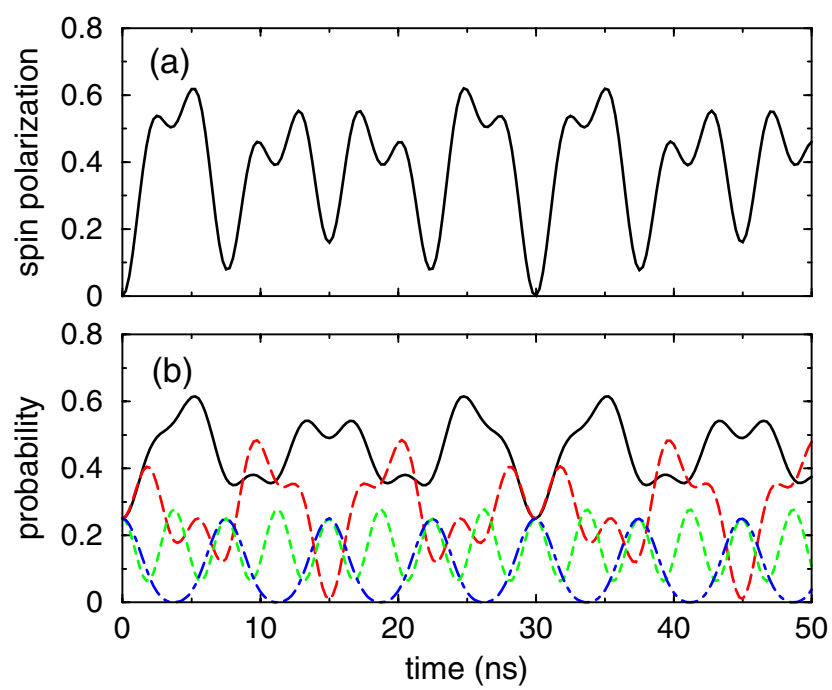

FIG. 4 (color online). Variation of the (a) degree of nuclear spin polarization and (b) probability in each nuclear spin state as a function of time for the ${ }^{37} \mathrm{Ca} 4 s^{2}{ }^{1} S_{0}-4 s 6 p{ }^{1} P_{1}(I=3 / 2)$ system under the application of a $3 \mathrm{kV} / \mathrm{cm}$ static electric field. In graph (b), solid, dot-dashed, long dashed, and dashed lines represent probabilities in the $m_{I}= \pm 3 / 2$ and $m_{I}= \pm 1 / 2$ nuclear spin states, respectively.

our method relies on the transient dynamics. A simple and obvious way is to remove two outmost valence electrons by intense ultrafast laser pulses at the moment when the nuclear spin polarization has reached the desired state. Upon the removal of the two valence electrons, nuclear spin is frozen, since the remaining electrons form a closed shell: Recall that the hyperfine coupling is a quantity proportional to $\mathbf{I} \cdot \mathbf{J}$, where $\mathbf{I}$ and $\mathbf{J}$ are the vector quantities of nuclear spin, $I$, and the total angular momentum, $J$, which is a sum of the orbital and spin angular momenta of electrons. Since $\mathbf{J}=\mathbf{0}$ for atoms or ions with closed shells, I automatically conserves its value, resulting in the freezing of nuclear spin. Note that the ultrafast removal of valence electrons does not deteriorate nuclear spin polarization, since hyperfine coupling does not take place during the removal of valence electrons. In order to find what conditions are required to remove the two valence electrons, we have carried out detailed calculations of ionization cross sections and find that the use of laser pulses at the wavelengths of, say, $\sim 266 \mathrm{~nm}$ or $\sim 200 \mathrm{~nm}$ with a peak intensity of $\sim 10^{13} \mathrm{~W} / \mathrm{cm}^{2}$ and a few hundred fs to a few hundred ps pulse duration, leads to the significant production of doubly charged ions of $\mathrm{Be}, \mathrm{Mg}$, and $\mathrm{Ca}$, etc., through the process of, for example, Ca $4 s 6 p{ }^{1} P_{1} \rightarrow$ $\mathrm{Ca}^{+} 3 s$ or $3 d \rightarrow \mathrm{Ca}^{2+}$. One of the immediate applications of nuclear spin-polarized ions produced this way is to trap them and perform $\beta$-NMR.
In conclusion, we have theoretically studied the dynamics of nuclear spin induced by short laser pulses. A rightcircularly polarized laser pulse creates a coherent superposition of hyperfine manifold, after which hyperfine coupling takes place. The time scale needed for nuclear spin polarization is inverse proportional to the energy splittings, which is inherently very slow $(\sim \mu \mathrm{s})$ due to the very small hyperfine energy intervals. This implies that the spontaneous decay will take place before sufficient nuclear spin polarization. In order to overcome this fundamental difficulty, we have introduced a static electric field, which results in the acceleration of hyperfine coupling by $2-3$ orders of magnitude. The physical mechanism of the acceleration is the state-dependent dc stark shifts. We have presented specific theoretical results for unstable isotopes, ${ }^{27} \mathrm{Mg}(I=1 / 2)$ and ${ }^{37} \mathrm{Ca}(I=3 / 2)$, which clearly demonstrated that nuclear spin polarization as high as $88 \%$ and $62 \%$, respectively, can be realized within the time scale of a few to tens of ns. After realizing nuclear spin polarization, nuclear spin polarization can be frozen by removing the valence electrons with the aid of the second short and intense laser pulse with the wavelengths of $\sim 266 \mathrm{~nm}$ or $\sim 200 \mathrm{~nm}$ easily obtained by the frequency tripling or quadrupling of Ti:sapphire lasers at $\sim 800 \mathrm{~nm}$. This is the fastest nuclear spin polarization ever achieved using an optical method, and it can be very effective not only for stable nuclei but also unstable nuclei with short lifetimes.

This work was supported by a Grant in Aid for scientific research from the Ministry of Education and Science of Japan.

*t-nakajima@iae.kyoto-u.ac.jp

[1] D. Fick, G. Grawert, and I. M. Turkiewicz, Phys. Rep. 214, 1 (1992).

[2] G. D. Cates, D. R. Benton, M. Gatzke, W. Happer, K. C. Hasson, and N. R. Newbury, Phys. Rev. Lett. 65, 2591 (1990).

[3] H. Reich and H. J. Jänsch, Nucl. Instrum. Methods Phys. Res., Sect. A 288, 349 (1990).

[4] A. N. Zelenskii, K. Jayamanna, C. D.P. Levy, M. McDonald, R. Ruegg, and P. W. Schmor, Nucl. Instrum. Methods Phys. Res., Sect. A 334, 285 (1993).

[5] J. Kessler, Polarized Electrons (Springer-Verlag, Berlin, 1985), 2nd ed.

[6] H. Winter, Phys. Rep. 367, 387 (2002).

[7] S. Iannotta, Atomic and Molecular Beam Methods, edited by G. Scoles (Oxford University, New York, 1988), p. 683.

[8] T. Nakajima, N. Yonekura, Y. Matsuo, T. Kobayashi, and Y. Fukuyama, Appl. Phys. Lett. 83, 2103 (2003).

[9] T. Nakajima, Appl. Phys. Lett. 84, 3786 (2004).

[10] T. Nakajima, Appl. Phys. Lett. 88, 111105 (2006).

[11] M. A. Bouchene, S. Zamith, and B. Girard, J. Phys. B 34, 1497 (2001). 